\title{
REDES NEURAIS ARTIFICIAIS APLICADAS NA PREVISÃO DO VTEC NO BRASIL
}

\author{
Artificial Neural Networks applied to VTEC prediction in Brazil \\ WAGNER CARRUPT MACHADO ${ }^{1}$ \\ EDVALDO SIMÕES DA FONSECA JUNIOR ${ }^{2}$ \\ ${ }^{1}$ Fundação Instituto Brasileiro de Geografia e Estatística UE-SC \\ ${ }^{1,2}$ Universidade de São Paulo-Escola Politécnica \\ Departamento de Engenharia de Transportes \\ Programa de Pós-graduação em Engenharia de Transportes \\ wagner@carrupt.com ; edvaldoj@usp.br
}

\begin{abstract}
RESUMO
Uma forma de se prever o conteúdo total de elétrons na direção vertical (VTEC Vertical Total Electron Content) usando a arquitetura de redes neurais artificiais (RNA) denominada de perceptrons de múltiplas camadas (MLP - MultipLayer Percetrons) é apresentada e avaliada nesta pesquisa. As entradas do modelo foram definidas como sendo a posição dos pontos ionosféricos (IPP - Ionospheric Pierce Point) e o tempo universal (TU), enquanto que a saída é o VTEC. As variações sazonais e de períodos mais longos são levadas em conta através da atualização do treinamento diariamente. Testes foram conduzidos sobre uma área que abrange o Brasil e sua vizinhança considerando períodos de alta e baixa atividade solar. As RNA foram treinadas utilizando informações dos mapas globais da ionosfera (GIM - Global Ionospheric Maps) produzidos pelo serviço internacional do GNSS (IGS International GNSS Service) das 72 horas anteriores à época de início da previsão. As RNA treinadas foram utilizadas para prever o VTEC por 72 horas ( $\mathrm{VTEC}_{\mathrm{RNA}}$ ).

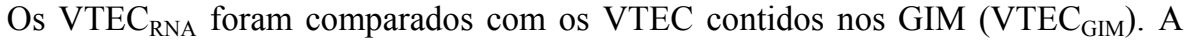
raiz do erro médio quadrático (RMS) da diferença entre o $\mathrm{VTEC}_{\mathrm{GIM}}$ e o VTEC $\mathrm{VTNA}_{\mathrm{R}}$ variou de 1,4 a 10,7 unidades de TEC (TECU). O erro relativo mostra que a RNA proposta foi capaz de prever o VTEC com 70 a $85 \%$ de acerto.
\end{abstract}

Palavras-chave: Redes Neurais Artificiais; Atraso Ionosférico; Previsão do VTEC.

\section{ABSTRACT}

An approach to predict vertical total electron content (VTEC) using multiplayer percetrons (MLP) artificial neural network (ANN) is presented. The models inputs 
were defined as being the ionospheric pierce point (IPP) positioning and the universal time (UT), while the output is the VTEC. The seasonal and longer period variations of the ionosphere were taken into account by daily training the ANN. Tests were conducted over an area covering Brazil and its vicinity in both high and low solar activity. The ANNs were trained using the previous 72 hours of data from the Global Ionospheric Maps (GIM) produced by the International GNSS Service (IGS). The trained ANNs were used to predict the VTEC to 72 hours $\left(\mathrm{VTEC}_{\mathrm{RNA}}\right)$. Comparisons between VTEC $_{\text {RNA }}$ and the VTEC contained into GIM (VTEC GIM $_{\text {) }}$ were made. The root mean square (RMS) of the differences between them varied from 1.4 to 10.7 TEC units (TECU). The relative error shows the ANN can correctly predict the VTEC $_{\text {GIM }}$ from 70 to $85 \%$.

Keywords: Artificial Neural Networks; Ionospheric Delay; Ionosphere.

\section{INTRODUÇÃo}

A passagem dos sinais GNSS através da ionosfera causa, dentre outros, o atraso ionosférico, o qual pode ser reduzido através de modelos matemáticos ou técnicas de posicionamento.

Os efeitos de primeira ordem provocados por esta camada da atmosfera são responsáveis por mais de $99 \%$ do referido atraso, e são proporcionais à quantidade total de elétrons (TEC - Total Electron Content) e inversamente proporcional ao quadrado da frequência do sinal (PETIT; LUZUM, 2010).

A magnitude do TEC está relacionada com a densidade de elétrons livres na ionosfera. Esta última está relacionada com a atividade solar, a posição geográfica, a hora local, a estação do ano e a condição geomagnética (DAVIES, 1990; SEEBER, 2003).

Destaca-se que, uma vez conhecido o TEC, o atraso ionosférico de primeira ordem pode ser calculado para dados GNSS de qualquer frequência. A magnitude do atraso causado por 1 unidade de TEC (TECU, onde 1 TECU $=1 \times 10^{16} \mathrm{el} / \mathrm{m}^{2}$ ), na fase da onda portadora das 3 frequências civis do GPS é de $-0,163 \mathrm{~m},-0,267 \mathrm{~m}$ e $0,291 \mathrm{~m}$, respectivamente.

Devido ao fato da ionosfera ser um meio dispersivo, é possível eliminar os efeitos de primeira ordem das observações GNSS com a utilização da combinação linear entre observações de duas frequências conhecida como livre da ionosfera (ion-free).

Visando melhorar o posicionamento de usuários de receptores de uma frequência, modelos regionais/globais da ionosfera, baseados em dados de redes de estações GNSS equipadas com receptores de dupla frequência, foram desenvolvidos (KOMJATHY, 1997; FELTENS, 1998; SCHAER, 1999; CAMARGO, 1999; COLOMBO et al., 2002; LIU, 2004).

É importante destacar que o serviço internacional do GNSS (IGS International GNSS Service) disponibiliza mapas globais da ionosfera (GIM Global Ionospheric Maps) no formato IONEX (IONphere maps Exchange format) 
desde 1998. Tais mapas são resultantes da combinação da solução de 4 centros de análise e possuem acuracidade que varia de 2 a 9 TECU (SCHAER; GURTNER e FELTENS, 1998; HERNADEZ-PAJAREZ et al., 2009, IGS, 2013). Uma avaliação deste produto do IGS no posicionamento absoluto com dados da estação da Rede Brasileira de Monitoramento Contínuo dos satélites GNSS (RBMC) localizada em Presidente Prudente em uma época de alta atividade solar foi realizada, a qual revelou uma melhora na acuracidade altimétrica e planimétrica de 76 e $26 \%$, respectivamente (MATSUOKA e CAMARGO, 2007).

Dados das redes GNSS também vêem sendo utilizados para modelar a ionosfera regionalmente, visando a geração de estações de referência virtuais ou parâmetros de correção válidos para uma dada região (FKP - Flaechen-KorrekturParameter). Tais soluções são discutidas em Fotopoulos e Cannon (2001), Dai et al. (2001) e Alves (2008).

Modelos empíricos, tais como o modelo de Klobuchar, o International Reference Ionosphere (IRI), o Low-latitude IONosphere model (LION), o Parameterized Ionospheric Model (PIM) e o Sheffield University Plamasphere Ionosphere Model (SUPIM), também permitem calcular/prever o TEC (KLOBUCHAR, 1987; DANIELL et al., 1995; BAILEY; BALAN; SU, 1997; BILITZA; REINISCH, 2007; BITTENCOURT et al., 2007). Dentre os modelos empíricos citados destaca-se o modelo desenvolvido por Klobuchar (1987), cujos coeficientes são enviados através da mensagem de navegação dos satélites GPS. Uma avaliação deste modelo durante alta atividade solar mostrou que ele reduziu em cerca de $53 \%$ os efeitos da ionosfera na pseudodistância da portadora L1 na estação da Rede Brasileira de Monitoramento Contínuo dos satélites GNSS (RBMC) localizada em Presidente Prudente (AGUIAR et al., 2003).

Outra forma de se calcular ou prever o TEC é utilizar redes neurais artificiais. Neste sentido algumas pesquisas foram publicadas nos últimos anos (CANDER et al., 1998; TULUNAY et al., 2006; HABARULEMA, MCKINNELL e CILLIERS, 2007; LEANDRO e SANTOS, 2007 e ACHARYA et al., 2011). Neste caso, a maioria dos modelos foi desenvolvida para prever o TEC por até 1 hora ou não permite a previsão de forma espacial.

Esta pesquisa apresenta uma solução recursiva para a previsão do VTEC utilizando MLP (MultiLayer Preceptorns) com uma única camada de 16 neurônios ocultos. Esta solução requer o conhecimento da série temporal do VTEC referenciado à posição do ponto ionosférico (IPP) das 72 horas anteriores à época de início das previsões. As variações sazonais e devido à atividade solar são levadas em conta através da atualização da solução a cada 24 horas.

A solução foi avaliada em uma área que abrange o Brasil e sua vizinhança durante alta e baixa atividade solar. Mapas globais da ionosfera produzidos pelo IGS foram utilizados como fonte de VTEC tanto no treinamento das RNA como na avaliação da solução.

Salienta-se que a previsão do VTEC, além de permitir que os efeitos de primeira ordem da ionosfera sobre o posicionamento com GNSS sejam reduzidos 
em tempo real, também pode ser utilizada em estudos que envolvem comunicação por ondas de rádio e clima espacial.

\section{REDES NEURAIS ARTIFICIAIS}

Redes neurais artificiais são sistemas inspirados nas estruturas de armazenamento e processamento do sistema nervoso biológico. Utilizando processamento paralelo, as RNA podem armazenar e disponibilizar conhecimento experimental. Elas são compostas por uma determinada quantidade de entradas e unidades de processamento, denominados de neurônios, os quais são ligados através de pesos sinápticos (HAYKIN, 1999).

As entradas são propagadas através da topologia da RNA, sendo transformadas pelos pesos sinápticos e pela função de ativação $(A F)$ dos neurônios. Recebendo entradas de $n$ neurônios $\left(y_{i}\right)$, o neurônio $k$ calcula a sua saída através de:

$$
y_{k}=A F\left(\sum_{i=1}^{n}\left(y_{i} w_{k i}\right)+b_{k}\right)
$$

onde $y_{i}$ é a saída calculada pelo neurônio $i, w_{k i}$ representa o peso sináptico entre o neurônio $i$ e o neurônio $k \mathrm{e} b_{k}$ é o peso entre um valor constante e diferente de zero ao neurônio $k$, conhecido como bias. Se o neurônio estiver ligado às entradas, o termo $y_{i}$ é substituído pela entrada correspondente.

Verifica-se que é necessário determinar os pesos sinápticos e bias para se utilizar uma RNA. A estimação de tais parâmetros, denominada de treinamento, se constitui de um processo iterativo onde os parâmetros iniciais são atualizados até a convergência do processo. Considerando a iteração $j$, o peso $w_{k i}$ é atualizado através de:

$$
w(j)_{k i}=w(j-1)_{k i}+\Delta w(j)_{i}
$$

com $w(j)_{i}$ sendo o vetor de correção ao parâmetro $w_{k i}$ na iteração $j$.

Funções de ativação não lineares do tipo sigmoidal antissimétrica são indicadas na solução de problemas de natureza não linear. Uma função que se enquadra nesta categoria é a denominada nesta pesquisa de bipolar, a qual é dada por (WILAMOWSKI et al., 2001):

$$
A F(x)=\frac{2}{1+e^{-x}}-1
$$

onde $x$ é calculado por: 


$$
x=\sum_{i=1}^{n}\left(y_{i} w_{k i}\right)+b_{k}
$$

A figura 1 traz o comportamento da função bipolar em função de $x$, mostrando que ela se torna saturada quando o valor desta variável é menor que -5 ou maior que +5 .

Figura 1 - Comportamento da função de ativação do tipo bipolar.

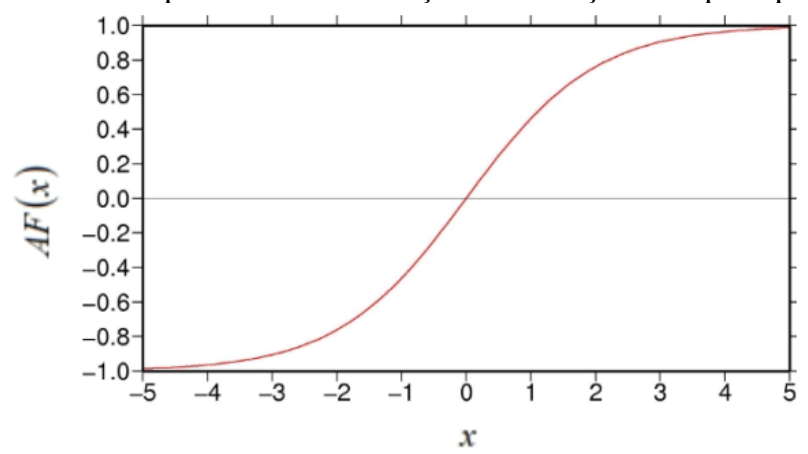

\subsection{Percéptrons de múltiplas camadas (MLP)}

Esta arquitetura de RNA também é conhecida como rede alimentada adiante de múltiplas camadas, devido ao fluxo da informação ser unidirecional. As entradas são propagadas da camada de entrada para a camada de saída, passando por uma ou mais camadas ocultas. A figura 2 mostra um exemplo desta arquitetura com duas entradas, uma camada oculta contendo dois neurônios e uma camada de saída constituída por apenas um neurônio.

Figura 2 - Exemplo da arquitetura MLP.

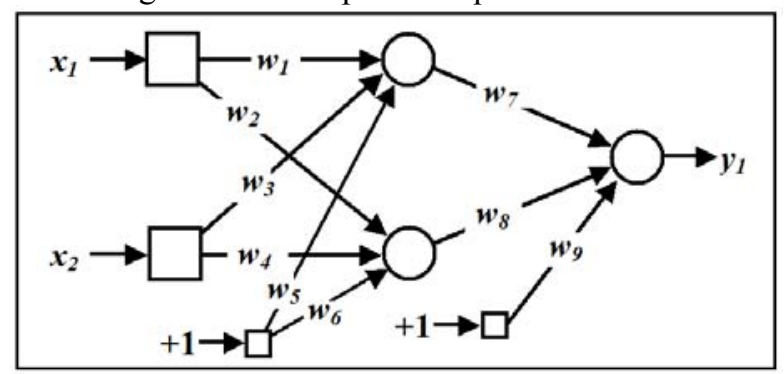

Cybenko (1989), Funahashi (1989) e Hornik; Stinchcombe e White (1989) mostraram que uma rede MLP com apenas uma camada oculta é capaz de 
aproximar qualquer função contínua dentro de uma precisão pré-estabelecida, desde que a camada oculta possua quantidade adequada de neurônios. Portanto, o desafio é determinar a quantidade de neurônios ideal.

Uma estratégia a se utilizar na definição da quantidade de neurônios ideal é a que considera uma MLP com a menor quantidade de neurônios possível sem afetar negativamente a sua capacidade de calcular a quantidade de interesse corretamente a partir de entradas que não foram utilizadas durante o treinamento, conhecida como generalização. Nesta situação, diz-se que o controle da complexidade do modelo é obtido através da regularização estrutural (BISHOP, 2008). A correlação-cruzada é uma ferramenta que pode ser utilizada na determinação da quantidade de neurônios ocultos ideal (STONE, 1978). Neste caso, uma parte dos dados de treinamento é separada para se realizar uma avaliação da solução. Treina-se RNAs com quantidade de neurônios diferentes e se selecionada a que produz o melhor resultado com os dados de avaliação (BISHOP, 2008).

$\mathrm{O}$ treinamento da MLP requer a disponibilidade de um conjunto de dados contendo entradas e saídas conhecidas (amostras). O objetivo do método de treinamento é minimizar uma função de custo calculada em função da diferença entre a saída calculada pela MLP e a saída correspondente do conjunto de dados de treinamento (HAYKIN, 1999). Após o treinamento, os parâmetros da MLP são fixos e a quantidade de interesse pode ser calculada.

Dentre os métodos desenvolvidos para treinar MLP, o de LevenbergMarquardt (LM) se destaca devido ao fato de combinar a estabilidade dos métodos baseados no gradiente descendente com a melhor taxa de convergência dos métodos de $2^{\mathrm{a}}$ ordem (BATTITI, 1992, WILAMOWSKI et al., 2001).

Considerando uma MLP com $p$ parâmetros a serem estimados com o método LM sendo treinada com $s$ amostras, o vetor de correção aos parâmetros na iteração $j$ é calculado através de (HAGAN e MENHAJ, 1994):

$$
\Delta w(j)=-\left[A^{T}(j) A(j) \eta(j) I\right]^{-1} A^{T}(j) e(j)
$$

onde:

$A$ - $(s \times p)$ matriz jacobiana;

$I$ - $(p \times p)$ matriz identidade;

$\eta$ - taxa de aprendizado;

$e-(s \times 1)$ vetor de erro (diferença entre a saída calculada pela MLP e a saída correspondente do conjunto de dados de treinamento).

Os elementos que compõem a matriz $A$ são definidos com detalhes em Hagan e Menhaj (1994) e Wilamowski et al. (2001). Uma forma otimizada para se calcular os elementos desta matriz, denominada de LM sem retropropagação, é apresentada em Wilamowski e Yu (2010).

$\mathrm{O}$ vetor de erro é calculado por: 


$$
e_{i, s}=d_{s}-y_{i, s}
$$

com $d$ sendo a saída do conjunto de dados de treinamento.

Neste método de treinamento, a taxa de aprendizado é ajustada automaticamente a cada iteração, assegurando a convergência do processo. Quanto menor a taxa de aprendizado, mais o processo se aproxima do método de GaussNewton. Caso contrário, ele se aproxima do método do gradiente descendente (HAGAN e MENHAJ, 1994).

Tendo em vista que a matriz a ser invertida é de ordem igual à quantidade de parâmetros a serem estimados, o custo computacional aumenta exponencialmente conforme a quantidade de tais parâmetros aumenta, sendo um fator a ser considerado na adoção deste método.

\section{MODELO PROPOSTO}

Nesta pesquisa, um modelo de previsão do VTEC baseado na arquitetura MLP com apenas uma camada de 16 neurônios ocultos foi desenvolvido e avaliado. A quantidade de neurônios da camada oculta foi determinada visando melhor generalização da RNA, onde a correlação cruzada foi empregada visando controlar a complexidade do modelo.

No modelo proposto, as entradas da RNA foram definidas como sendo a posição do IPP e o TU, conforme ilustra a figura 3 .

Figura 3- Modelo de rede neural artificial proposto.

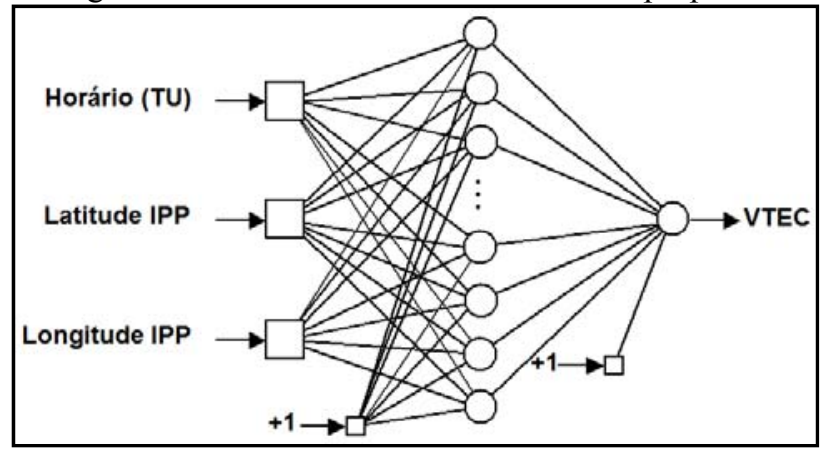

Os conjuntos de dados utilizados no treinamento e na avaliação das RNA foram definidos como sendo a série temporal da grade de VTEC das 72 horas anteriores e posteriores ao início da previsão, respectivamente. Os GIM do IGS foram delimitados entre as latitudes $+15^{\circ}$ e $-40^{\circ}$ e as longitudes $-30^{\circ}$ e $-85^{\circ}$, resultando em 39 grades, com intervalo de duas horas, com 276 vértices cada uma (10.764 amostras). A figura 4 ilustra a grade de VTEC utilizada no treinamento das RNA. 
Figura 4 - Grade de VTEC do modelo.

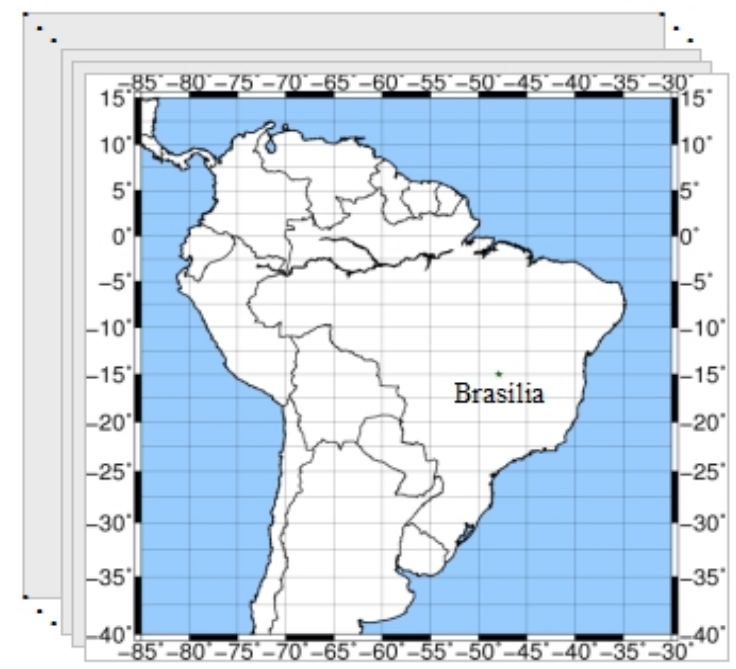

Os valores de interesse são lidos dos arquivos dos GIM, no formato IONEX. As entradas do modelo são normalizadas através da divisão da diferença entre a entrada e a sua média pelo desvio-padrão correspondente. Este procedimento visa acelerar o processo de aprendizado e diminuir a probabilidade do algoritmo convergir para um mínimo local. Um programa para extrair as informações de interesse dos GIM, executar o pré-processamento das entradas e escrever os arquivos dos conjuntos de estimação e validação no formato do programa utilizado no treinamento foi desenvolvido durante a pesquisa.

Para treinar as RNA, foi desenvolvido um programa que emprega o método LM sem retropropagação, onde o treinamento da RNA é finalizado quando o número de iterações for igual a 200.

As variações da ionosfera provocadas pela atividade solar e a estação do ano foram levadas em consideração através da atualização dos parâmetros da MLP a cada 24 horas. No primeiro dia de treinamento, os parâmetros foram iniciados com valores randômicos entre -1 e +1 . Nos demais dias, os parâmetros estimados no dia anterior foram utilizados.

A função de ativação dos neurônios da camada oculta foi definida como sendo a do tipo bipolar (eq. 3), enquanto a do neurônio da camada de saída foi do tipo linear. Com esta prática, evitou-se de re-escalar a saída, bem como a saturação do neurônio de saída. Por conseguinte, além dos resultados poderem ser utilizados diretamente, a convergência do processo é acelerada.

\section{EXPERIMENTOS}

$\mathrm{Na}$ avaliação da solução proposta, foram treinadas RNA com dados dos GIM 
de 21 de março a 4 de abril (dias 80 a 94) de 2001 e de 16 a 30 de junho (dias 167 a 181) de 2009. As RNA treinadas foram utilizadas para prever 72 horas do VTEC para a grade apresentada na figura 4. As previsões foram comparadas com os VTEC contidos nos GIM correspondentes, permitindo que análises acerca das diferenças entre o $\mathrm{VTEC}_{\mathrm{RNA}}$ e o $\mathrm{VTEC}_{\mathrm{GIM}}$ fossem conduzidas. O erro relativo proposto por Leandro e Santos (2007) também foi investigado. Observa-se que dados de 18 dias do último período de máxima atividade solar, incluindo uma tempestade geomagnética severa, e de 18 dias do último período de mínima atividade solar foram considerados.

Visando a regularização estrutural da RNA, a quantidade de neurônios da camada oculta foi determinada através da comparação da raiz do erro médio quadrático (RMS - Root Mean Square) do conjunto de validação produzida por RNA treinadas com quantidades diferentes de neurônios ocultos para os dias 21/03/2001 (alta atividade solar), 30/03/2001 (tempestade geomagnética) e 19/06/2009 (baixa atividade solar).

Como a atividade solar é o fator que mais influencia na quantidade de elétrons livres, convém mensurá-la. Os valores do fluxo solar 10,7 cm medidos na estação de Penticton, Canadá, obtidos do centro de dados geofísicos do National Oceanic and Atmospheric Administration (NOAA, 2012), são mostrados na figura 5.

Figura 5 - Histórico do fluxo solar 10,7 cm na estação Penticton.

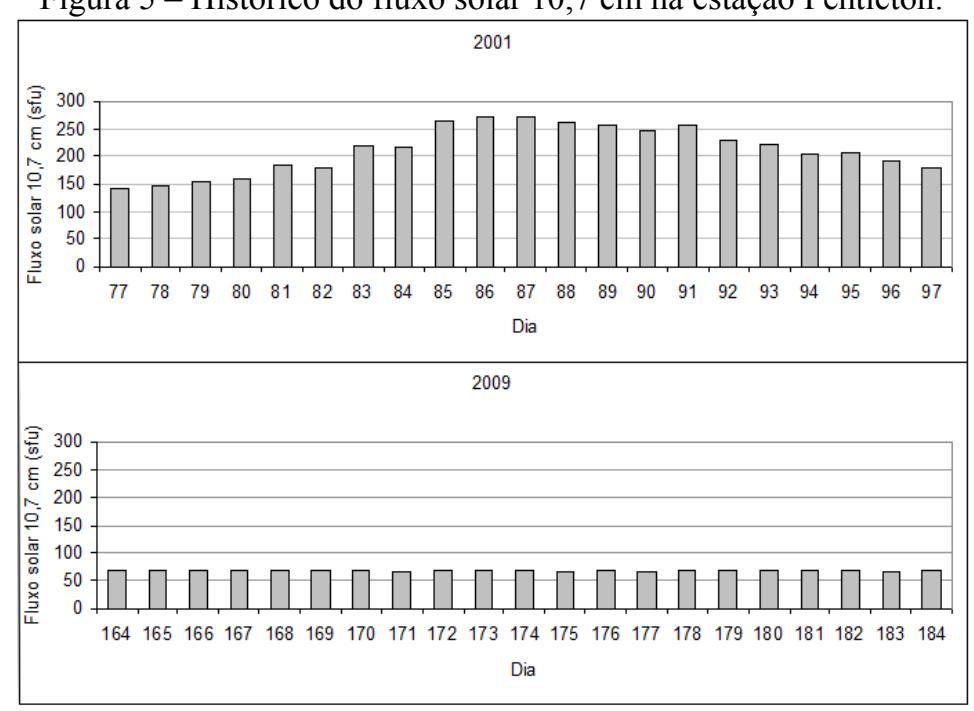

Durante o período de alta atividade solar considerado nesta pesquisa, a medida do fluxo solar $10,7 \mathrm{~cm}$ realizada na estação Penticton variou de 139,8 a 273,5 unidades de fluxo solar (sfu - solar flux units), apresentando valor médio de cerca 
de 212,6 sfu. No período de baixa atividade solar, esta grandeza variou de 66,5 a $68,5 \mathrm{sfu}$, com média de $67,5 \mathrm{sfu}$.

Outra grandeza de interesse a ser mensurada é a condição geomagnética, visto que ela está relacionada com variações de curto período na densidade de elétrons livres. A figura 6 mostra o histórico do índice Dst, obtido do serviço de distribuição de dados para fins de estudos relacionados com o campo geomagnético da Universidade de Kyoto (KYOTO UNIVERSITY, 2012).

Figura 6 - Comportamento do índice Dst nos dois períodos.

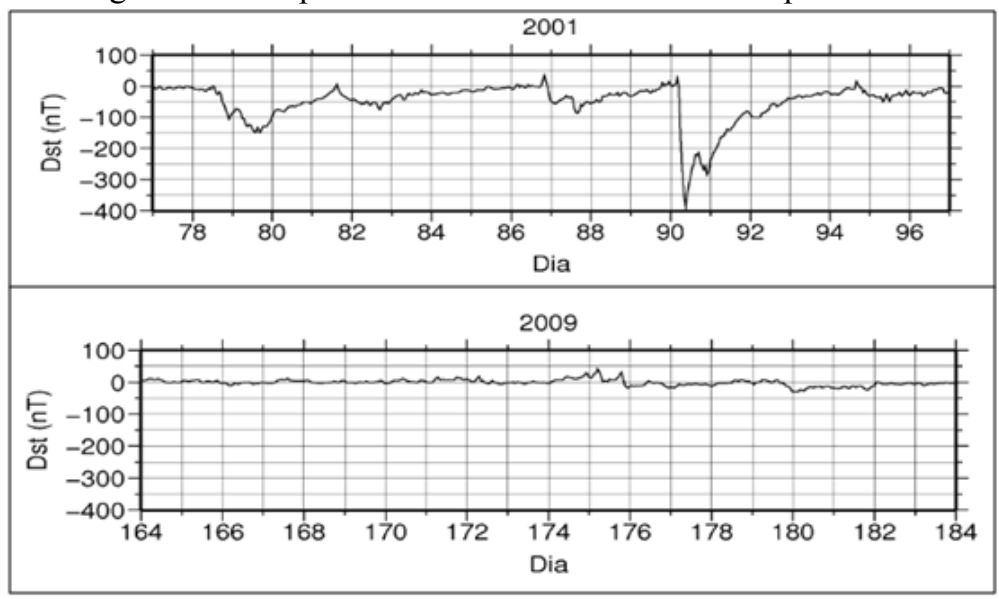

Observa-se que no período de alta atividade solar, o índice Dst apresentou variações maiores do que no período de baixa atividade solar, atingindo o valor de $-400 \mathrm{nT}$ no dia 90 (29/03/2001), quando ocorreu a tempestade geomagnética. Durante o período de baixa atividade solar este índice não chegou a $-50 \mathrm{nT}$.

Nas análises, quatro casos foram considerados:

(a) caso 1: período de alta atividade solar sem influência de tempestade geomagnética;

(b) caso 2: previsão para o dia em que a tempestade geomagnética ocorreu;

(c) caso 3: período em que a previsão do VTEC foi realizada utilizando dados influenciados pela tempestade geomagnética e o

(d) caso 4 : período de baixa atividade solar.

\section{RESULTADOS E ANÁLISES}

Embora o treinamento tenha sido encerrado com 200 iterações, verificou-se que a convergência do processo foi alcançada com menos de 55 iterações. É importante destacar que o tempo de processamento das 200 iterações foi de aproximadamente 2,5 min. Além disso, verificou-se que iniciar os parâmetros a serem estimados com os parâmetros estimados no dia anterior o "conhecimento" 
adquirido pela RNA foi transmitido e a convergência do processo foi atingida com 5 iterações.

A figura 7 mostra os valores do $\mathrm{VTEC}_{\mathrm{GIM}}$ e do $\mathrm{VTEC}_{\mathrm{RNA}}$ para todas as coordenadas da grade durante os dois períodos do experimento

Figura $7-\mathrm{VTEC}_{\mathrm{GIM}} \mathrm{x} \mathrm{VTEC}_{\mathrm{RNA}}$.

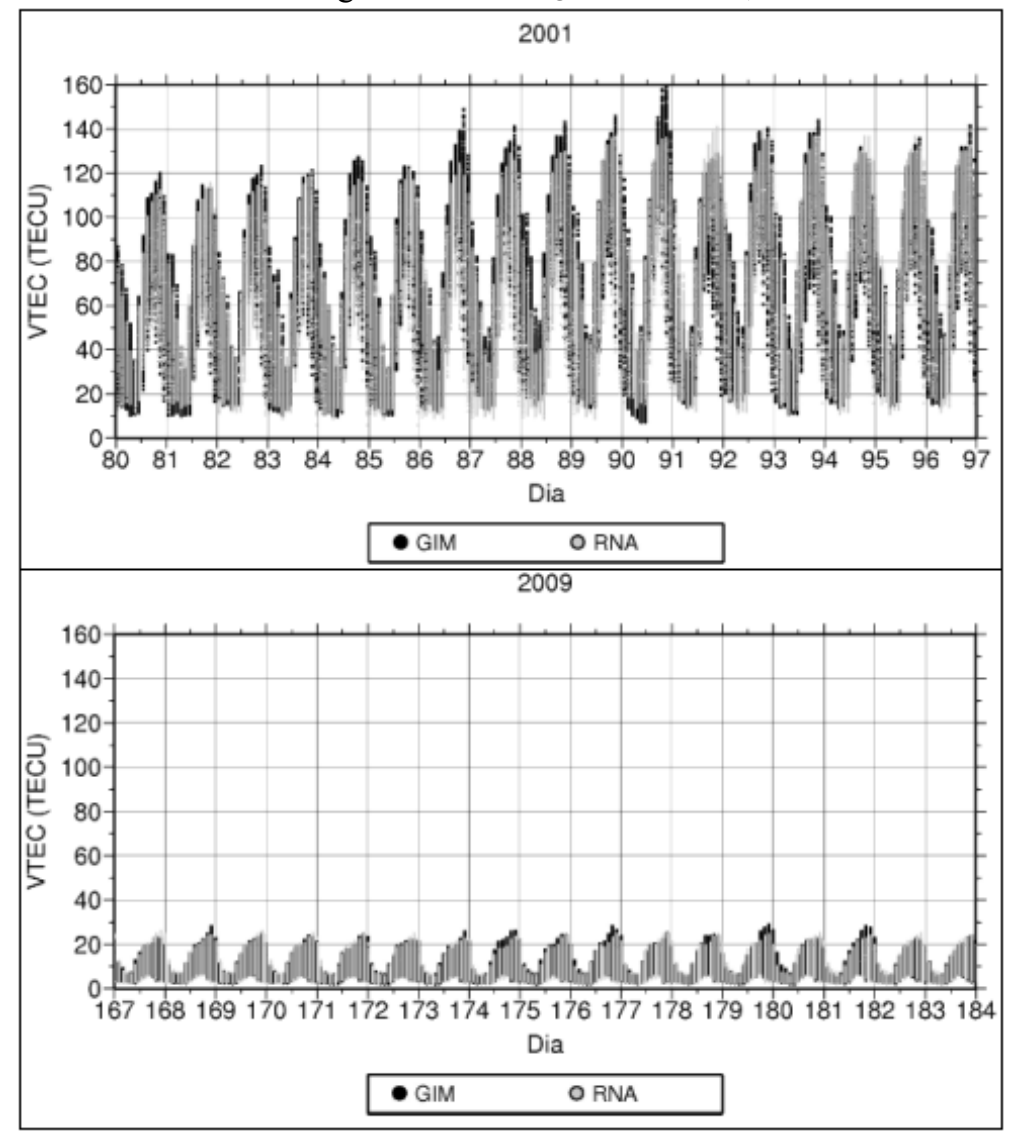

Verifica-se que os valores máximos do $\mathrm{VTEC}_{\mathrm{GIM}}$ durante alta atividade solar são cerca de 8 vezes maiores do que em baixa atividade solar e que ocorrem variações de curto período em ambos os períodos. Comparando o $\mathrm{VTEC}_{\mathrm{GIM}}$ com o VTEC $_{\text {RNA }}$ observa-se que a RNA é auto-adaptativa. Porém, como os parâmetros da MLP são calculados com dados das 72 horas anteriores, a RNA não é capaz de se adaptar às variações de períodos mais curtos do $\mathrm{VTEC}_{\mathrm{GIM}}$, como pode ser visto do dia 89 para o 90 de 2001 e do dia 179 para o 180 de 2009. 
As dispersões entre VTEC $_{\mathrm{GIM}}$ e $\mathrm{VTEC}_{\mathrm{RNA}}$ para os quatro casos foram investigadas e os coeficientes de correlação foram calculados, como pode ser visto na figura 8. É importante notar que a escala do gráfico do caso 4 é diferente dos demais para que fosse possível visualizar a dispersão obtida durante o período de baixa atividade solar com mais detalhes.

Figura 8 - Dispersão entre o VTEC $\mathrm{VIIM}_{\mathrm{M}}$ e o VTEC $\mathrm{VNNA}_{\mathrm{R}}$.

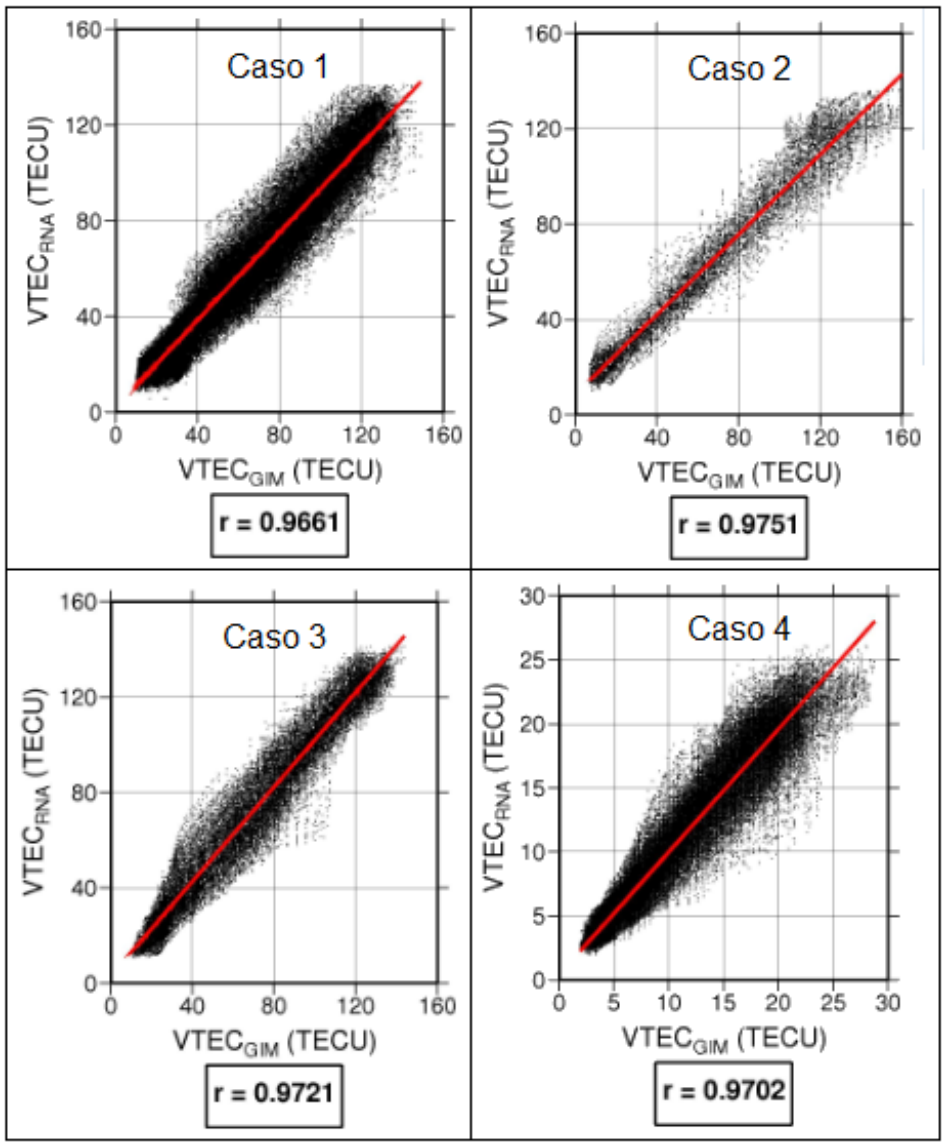

Observa-se, na figura 8, que a dispersão tende a aumentar proporcionalmente ao valor do VTEC até a aproximadamente 50 TECU e depois disso a dispersão se estabiliza. Um comportamento diferente pode ser visto no caso 3, onde a dispersão aumentou até aproximadamente 100 TECU e depois diminuiu até um determinado patamar. $\mathrm{O}$ coeficiente de correlação (r) revela uma forte correlação entre $\mathrm{o}$ VTEC $_{\text {GIM }}$ e o VTEC RNA $_{\text {. }}$ 
A diferença entre o VTEC $_{\text {GIM }}$ e o $\operatorname{VTEC}_{\text {RNA }}$ (dVTEC) durante os dois períodos foi analisada e seu comportamento é apresentado na figura 9.

Figura 9 - Comportamento da diferença entre o VTEC GIM $_{\text {e o VTEC }}$ RNA (dVTEC).

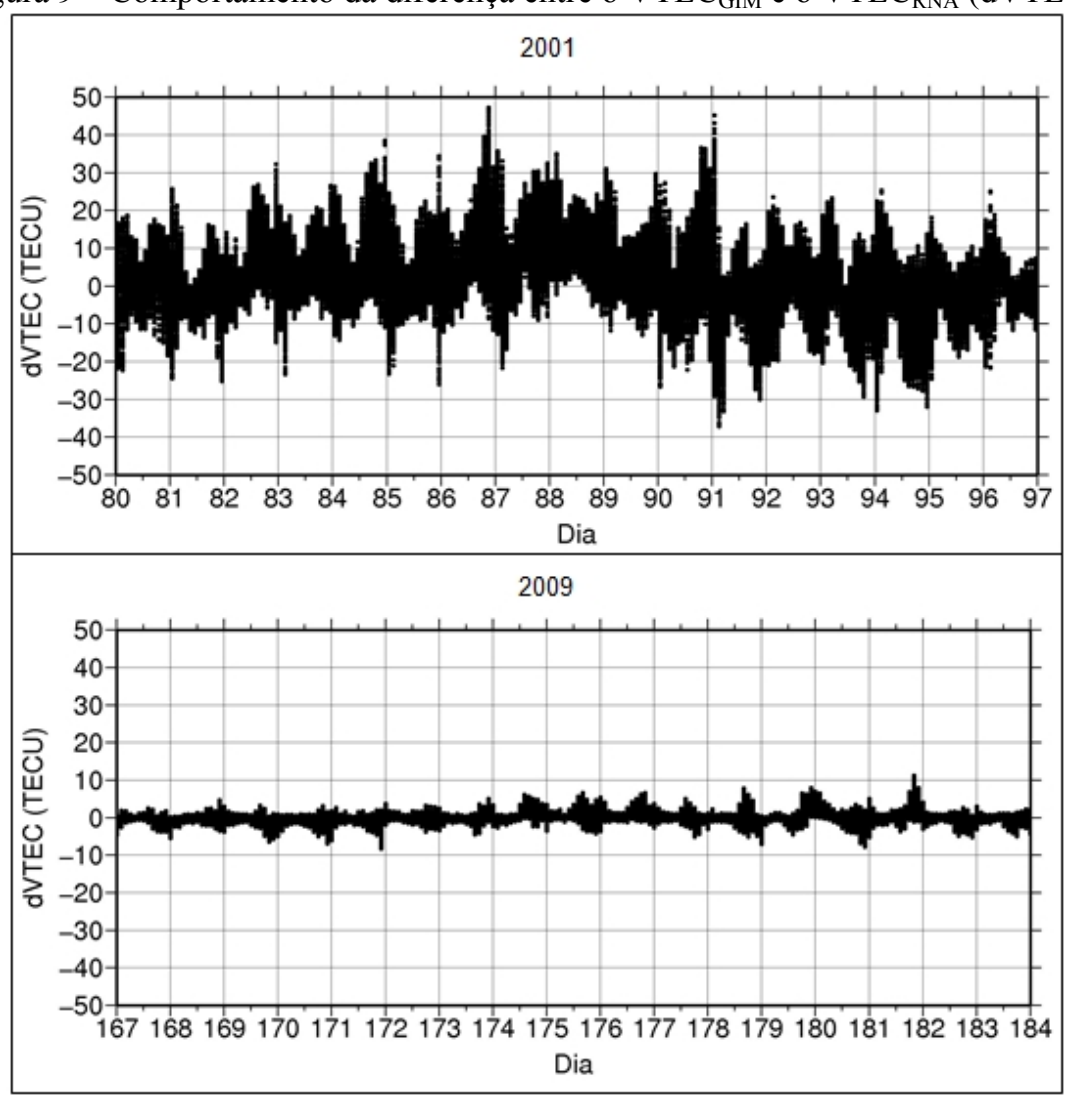

Constata-se que a dVTEC possui um comportamento cíclico em ambos os períodos, onde os valores máximos são atingidos quanto mais próximo dos valores máximos diários do VTEC.

A distribuição da dVTEC para os quatro casos pode ser vista na figura 10 , verificando-se que para os casos 1 e 3 , cerca de $88 \%$ das dVTEC ficaram entre \pm 015 TECU. No caso 2, esta porcentagem diminui de aproximadamente dois pontos percentuais. Para o caso $4,99 \%$ das diferenças ficaram entre \pm 5 TECU. 
Figura 10 - Distribuição da diferença entre o VTEC $_{\text {GIM }}$ e o VTEC $\mathrm{VNNA}_{\text {. }}$

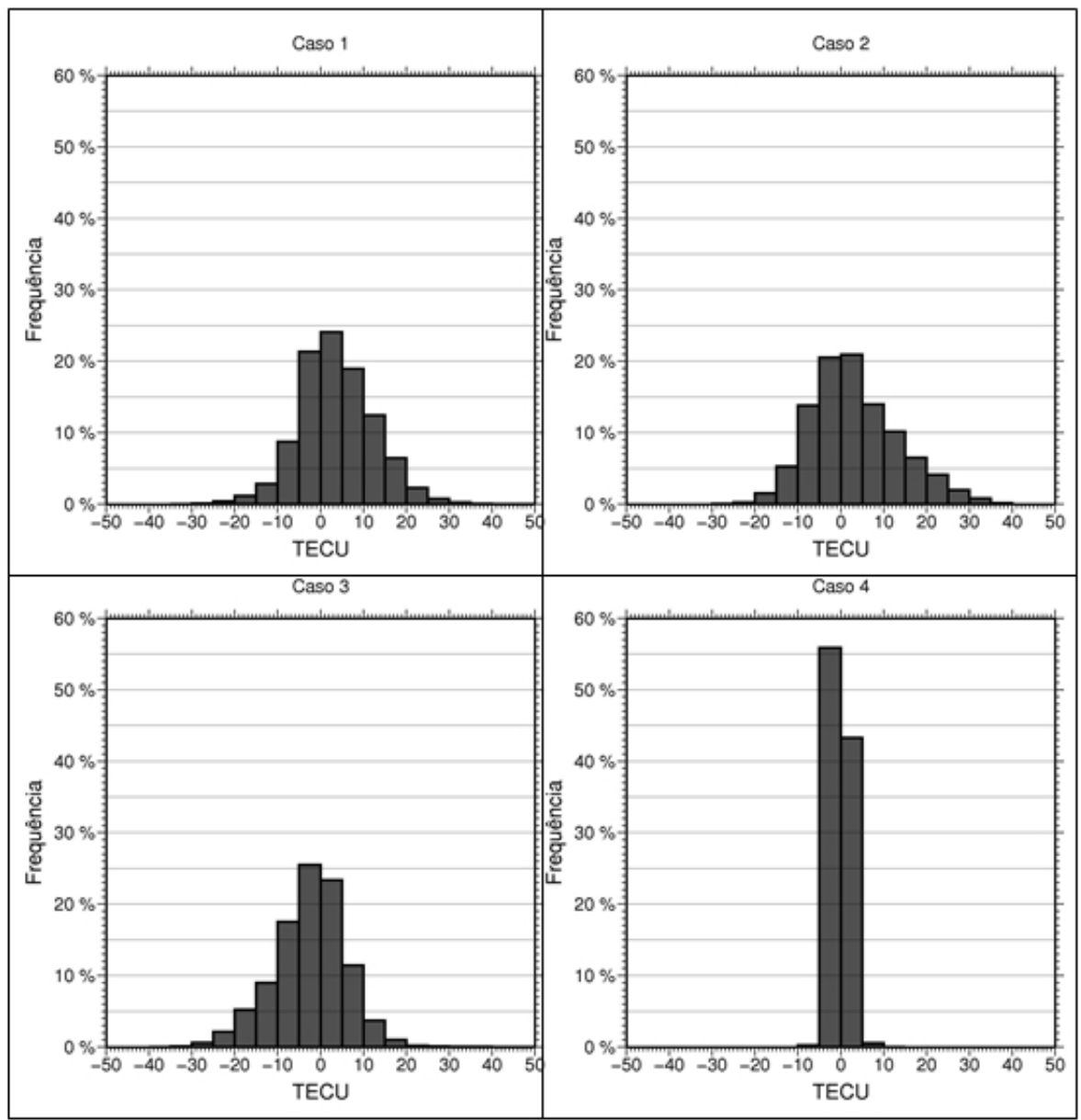

A tabela 1 contém os valores máximos, médios e mínimos, bem como os RMS e os desvios-padrão da dVTEC considerando todos os resultados para os quatro casos.

Tabela 1 - Estatísticas da diferença entre o VTEC $_{\text {GIM }}$ e o $\operatorname{VTEC}_{\mathrm{RNA}}$ (TECU).

\begin{tabular}{c|c|c|c|c|c}
\hline Caso & Max & Med & Min & RMS & Desvio-padrão \\
\hline 1 & 44,6 & 3,7 & $-34,2$ & 9,5 & 8,8 \\
\hline 2 & 38,4 & 3,2 & $-29,8$ & 10,7 & 10,2 \\
\hline 3 & 43,0 & $-2,5$ & $-35,0$ & 8,8 & 8,5 \\
\hline 4 & 11,1 & $-0,1$ & $-8,3$ & 1,4 & 1,4 \\
\hline
\end{tabular}

Bol. Ciênc. Geod., sec. Artigos, Curitiba, v. 19, nº 2, p.227-246, abr-jun, 2013. 
Observa-se que ocorreu uma situação na qual a dVTEC chegou a aproximadamente 47 TECU durante alta atividade solar, valor este reduzido para 11 TECU durante baixa atividade solar. Por outro lado, o RMS indica que estas diferenças devem permanecer na maior parte do tempo abaixo de 11 TECU durante alta atividade solar e de 1,5 TECU caso contrário. O atraso provocado por tais diferenças varia de 1,79 a $0,24 \mathrm{~m}$ em L1 e de 3,2 a 0,44 m em L5.

A figura 11 contém a variação diária do RMS relacionado com a dVTEC para os dois períodos considerados no experimento.

Figura 11 - Variação da RMS da diferença entre o VTEC $_{\mathrm{GIM}}$ e o VTEC $_{\mathrm{RNA}}$.

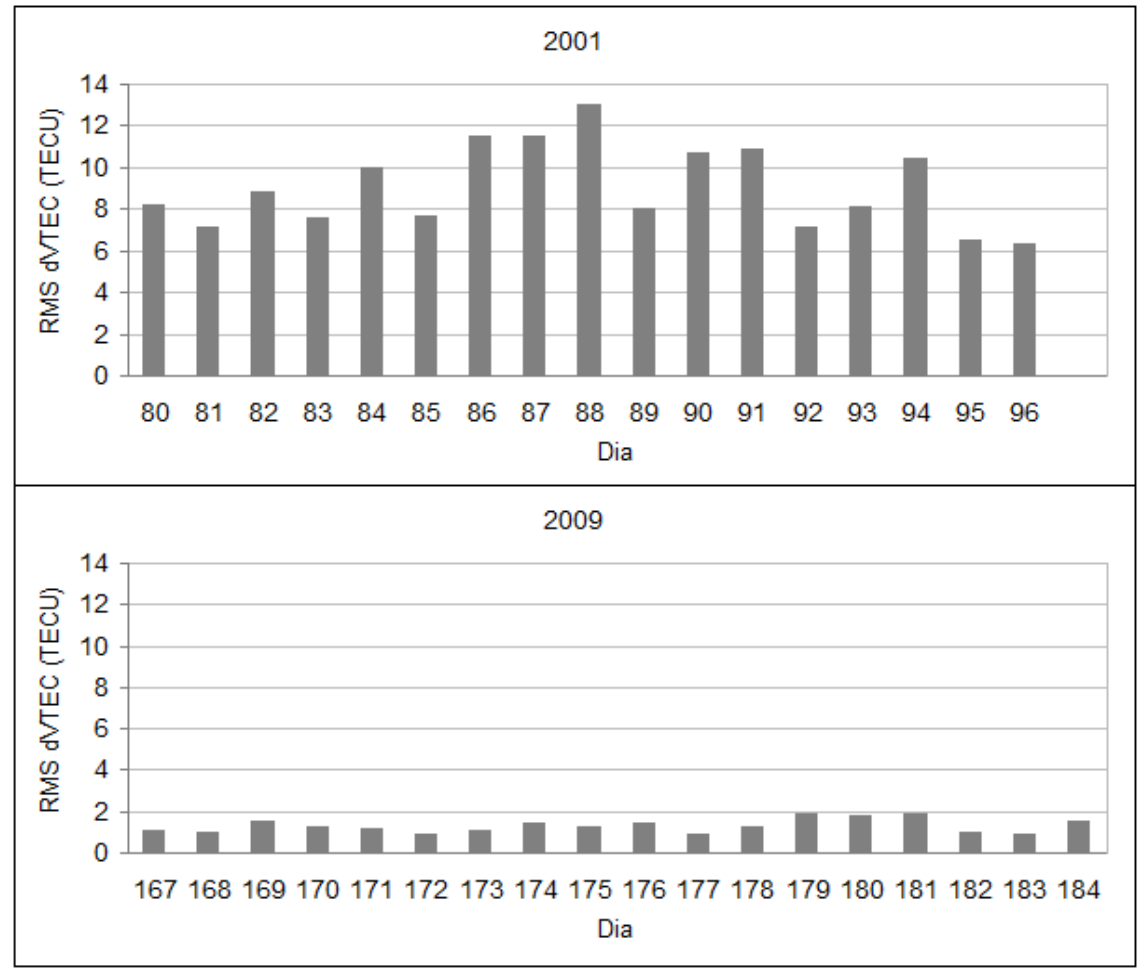

Verifica-se que durante alta atividade solar o valor do RMS é cerca de 5 a 12 vezes maior do que no período de baixa atividade solar. As variações da dVTEC são mais estáveis para o caso 4 (2009), variando de 1 a, aproximadamente, 2 TECU. Comparando-se a figura 11 com as figuras 5 e 6, observa-se a relação do valor do RMS com a atividade solar e com a condição geomagnética, justificando o pior resultado ter ocorrido para o dia 88 , dois dias antes da tempestade geomagnética. Além disso, também pode ser observado que a tempestade geomagnética não 
deteriora a solução de forma significativa.

Deve-se ressaltar, também, que em 2001 os arquivos dos GIM foram produzidos com dados de apenas 3 estações localizadas em território brasileiro, ao passo que em 2009, 10 estações foram utilizadas.

O erro relativo $(\varepsilon)$ foi analisado e sua série temporal pode ser vista na figura 12.

Figura 12 - Série temporal do erro relativo.

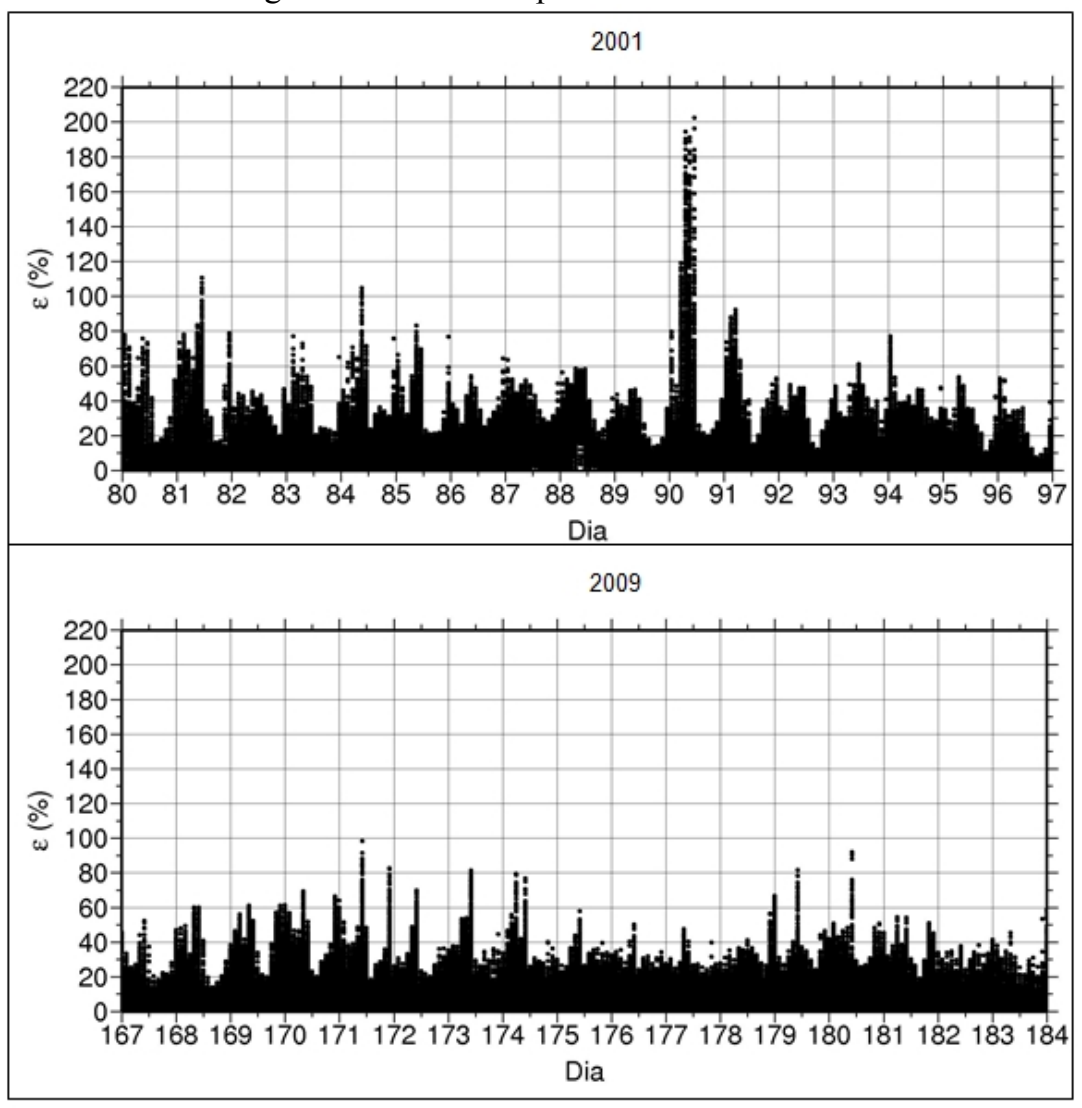

A figura 12 mostra que o erro relativo possui a mesma magnitude para ambos os períodos, variando de aproximadamente 0 a $20 \%$ durante maior parte do tempo. Uma exceção ocorreu no dia 90 (caso 2) onde tal erro alcançou mais de $200 \%$ entre as 2 e $5 \mathrm{~h}$ (horário local). Tal efeito ocorreu por que o valor do VTEC $_{\text {GIM }}$ sofreu um decréscimo devido à tempestade geomagnética. Além disso, um reflexo da tempestade geomagnética pode ser visto no dia 91, mas em uma escala menor. Os 
erros relativos dos dias 92 e 93 têm o mesmo nível dos erros obtidos no caso 1, mostrando que a utilização de dados afetados pela tempestade geomagnética não deteriora a predição do VTEC de forma significativa. A tabela 2 mostra os valores máximos, médios, mínimos, RMS e desvios-padrão do $\varepsilon$ para os quatro casos.

Tabela 2 - Estatísticas do erro relativo (\%).

\begin{tabular}{c|c|c|c|c|c}
\hline Caso & Max & Média & Min & RMS & Desvio-padrão \\
\hline 1 & 110,7 & 13,2 & 0,1 & 17,3 & 11,2 \\
\hline 2 & 202,4 & 17,1 & 0,1 & 30,0 & 24,7 \\
\hline 3 & 92,3 & 12,3 & 0,1 & 17,4 & 12,3 \\
\hline 4 & 98,4 & 11,3 & 0,1 & 14,8 & 9,6 \\
\hline
\end{tabular}

O RMS do $\varepsilon$ variou de $30 \%$ (caso 2) até aproximadamente $15 \%$ (caso 4), mostrando que a RNA teve a qualidade de sua predição degradada pela tempestade geomagnética, visto que a RMS permaneceu em torno de $17 \%$ nos casos 1 e 3 .

\section{CONSIDERAÇÕES E CONCLUSÕES}

Uma solução recursiva para prever o VTEC no Brasil por 72 horas utilizando MLP com uma única camada de 16 neurônios foi proposta e testada durante alta e baixa atividade solar, bem como durante uma tempestade geomagnética severa.

É importante destacar que a previsão do VTEC permite que os efeitos de primeira ordem da ionosfera sobre o posicionamento com GNSS sejam reduzidos em tempo real. Além disso, tal previsão pode ser utilizada em estudos que envolvem comunicação por ondas de rádio, geofísica espacial e clima espacial.

Os experimentos mostraram que esta solução é autoadaptativa. Além disso, constata-se que as previsões das RNA possuem o mesmo nível de acuracidade dos GIM divulgada pelo IGS, variando de 1,4 TECU, no período de baixa atividade solar, a 10,7 TECU, durante alta atividade solar com tempestade geomagnética. Por fim, o erro relativo mostrou que o $\mathrm{VTEC}_{\mathrm{GIM}}$ foi corretamente previsto de 70 a $85 \%$.

Destaca-se que, embora os GIM do IGS tenham sido utilizados nos experimentos, outras fontes de VTEC podem ser empregadas. Neste sentido, experimentos utilizando mapas do VTEC produzidos pelo Mod_Ion, desenvolvido na FCT/Unesp de Presidente Prudente, estão sendo realizados. Além disso, é possível realizar experimentos com os mapas ionosféricos regionais da América do Sul (SARIM - South American Regional Ionospheric Maps), produzidos pela Universidade de La Plata, Argentina, e disponibilizados no âmbito do projeto SIRGAS (Sistema de Referência Geocêntrico para as Américas).

Visando melhorar a qualidade das previsões, informações sobre a atividade solar e a condição geomagnética podem ser considerados no modelo em trabalhos futuros. Além disso, sugere-se que uma avaliação das pevisões no posicionamento seja conduzida. 


\section{AGRADECIMENTOS:}

O primeiro autor gostaria de agradecer ao IBGE, pela concessão de licença em tempo integral para realização desta pesquisa. Agradecemos também as contribuições dos revisores.

\section{REFERÊNCIAS BIBLIOGRÁFICAS}

ACHARYA, R.; ROY, B.; SIVARAMAN, M. R.; DASGUPTA, A. Prediction of ionospheric Total Electrons Content using adaptive neural network with in-situ learning algorithm. Advances in Space Research, v. 47, n. 1, p. 115-123, Jan., 2011.

AGUIAR, C. R. et al. Determinação do Erro Sistemático na Pseudodistância devido à ionosfera: Avaliação da performance do Mod_Ion e do Modelo de Klobuchar. In: UFPR. (Org.). Série em Ciências Geodésicas. Curitiba: Imprensa Universitária da UFPR, 2003. v. 3.

ALVES, D. B., M. Posicionamento GPS utilizando o conceito de estação virtual, 2008. 165 p., Tese (Doutorado) - Faculdade de Ciências e Tecnologia, Universidade Estadual Paulista, Presidente Prudente, 2008.

BAILEY, G. J; BALAN, N.; SU, Y. Z. The Sheffiled University plasmasphere ionosphere model - a review. Journal of Atmospheric Solar-Terrestrial Physics, v. 59, n. 13, p. 1541-1552, Sept., 1997.

BATTITI, R. First and second order methods for learning: between steepest descent and Newton's method. Neural Computation, v. 4, n. 2, p. 141-166, Mar.,1992.

BILITZA, D.; REINISCH, B. W. International Reference Ionosphere 2007: Improvements and new parameters. Advances on Space Research, v. 48, n. 2, p. 599-609, Aug., 2007.

BISHOP, C. M. Neural Networks for pattern recognition. Oxford: Oxford University Press, 2008. 482 p.

BITTENCOURT, J. A. et al. LION: a dynamic computer model for the low-latitude ionosphere. Annales Geophysicae, v. 25, p. 2371-2392, Nov., 2007.

CAMARGO, P. O. Modelo regional da ionosfera para uso em posicionamento com receptores GPS de uma freqüência. 1999. 191 p. Tese (Doutorado) - Setor de Ciências da Terra, Universidade Federal do Paraná, Curitiba, 1999.

CANDER, R. Lj.; MILOSAVLJEVIC, M. M.; STANKOVIC, S. S.; TOMASEVIC, S. Ionospheric forecasting technique by artifical neural network. Eletronics Letters, v. 34, n. 16, 1573-1574, Aug., 1998.

COLOMBO, O. L.; HERNANDEZ-PAJARES, M. JUAN, J. M.; SANZ, J. Widearea, carrier-phase ambiguity resolution using a tomographic model of the ionosphere. NAVIGATION: Journal of The Institute of Navigation, v. 49, n. 1, p. 61-69, 2002.

CYBENKO, G. Approximations by superpositions of sigmoidal functions. Math. Control Signal Systems, v. 2, p. 303-314, 1989. 
DANIELL, R. E., et al. Parameterized Ionosphere Model: A global ionospheric parameterization based on first principle models. Radio Science, v. 30, n. 5, p 1499-1510, 1995.

DAI, L., et al. A Study on GPS/GLONASS Multiple Reference Station Techniques for Precise Real-Time Carrier Phase-Based Positioning. In: ION GPS-2001, 2001. Salt Lake City. Proceedings..., 2001.

DAVIES, K. Ionospheric radio. London: IEE (Institute of Electrical Engineer), 1990. 580 p. (Eletromagnetic Waves Series v. 31).

FELTENS, J. Chapman Profile Approach for 3-d Global TEC Representation. In: IGS ANALYSIS CENTERS WORKSHOP, 1998. Darmstadt. Proceedings, 1998, p. 285-297.

FOTOPOULOS, G.; CANNON, M. E. An Overview of Multiple-Reference Station Methods for cm-Level Positioning. GPS Solutions, v. 4, n. 3, p. 1-10, Jan., 2001.

FUNAHASHI, K. On the approximate realization of continuous mappings by neural networks. Neural Networks, v. 2, n. 3, p. 183-192, 1989.

HABARULEMA, J. B.; McKINNEL, L. A.; CILLIERS, P. J. Prediction of GPS total electron content using neural network over South Africa. Journal of Atmospheric and Solar-Terrestrial Physics, v. 69, n.15, p. 1842-1850, Nov., 2007.

HAGAN, M. T. e MENHAJ, M. B. Training feedforward neural networks with the Marquardt algorithm. IEEE Transactions on Neural Networks, v. 5, n. 6, p. 989-993,1994.

HAYKIN, S. Redes neurais: princípios e prática. 2a ed. São Paulo: Prentice Hall, 1999. $900 \mathrm{p}$.

HERNÁNDEZ-PAJARES, M.; JUAN, J. M.; SANZ, J.; ORUS, R..; GARCÍARIGO, A.; FELTENS, J.; KOMJATHY, A.; SCHAER, S. C.; KRANKOWSKI, A. The IGS VTEC maps: a reliable source of ionospheric information since 1998. Journal of Geodesy, v. 83, n. 3-4, p. 263-275, Mar., 2009.

HORNIK, K.; STINCHCOMBE, M.; WHITE, H. Multilayer feedforward networks are universal approximators. Neural Networks, v. 12, p. 359-366, 1989.

IGS. IGS products, 2013. Disponível em: <http://www.igs.org/components/ prods.html> . Acesso em: 20 de mar. de 2013.

KLOBUCHAR J, A. Ionospheric Effects on GPS. In Parkinson B. W. e Spilker Jr. J .J. Global Positioning System: Theory and Applications, Vol. I, Cambridge American Institute of Aeronautics and Astronautics, v. 163, p. 485 - 515, 1996.

KLOBUCHAR J, A. Ionospheric time-delay algorithm for single-frequency GPS users. IEEE Transactions on Aerospace and Electronic Systems, v. 23,n. 3, p. 325-331, May, 1987.

KOMJATHY, A. Global ionospheric Total Electron Content mapping using Global Positioning System. 1997. 248 p. Tese (Doutorado) - Department of Geodesy and Geomatics Engineering. University of New Brunswick, Frederiction, 1997. 
KYOTO UNIVERSITY World data center for geomagnetism. Disponível em: $<$ http://wdc.kugi.kyoto-u.ac.jp/index.html>. Acesso em: 15 de maio 2012.

LEANDRO, R. F.; SANTOS, M. C. A neural network approach for regional vertical electron content modeling. Studia Geophysica et Geodaetica, v. 51, n. 2, p. 279-292, Apr., 2007.

LIU, G. G. Ionosphere tomographic modeling and applications using Global Positioning System (GPS) measurements. 2004. 304 p. Tese (Doutorado) Department of Geomatics Engineering, University of Calgary, Calgary, 2004.

MATSUOKA, M. T.; CAMARGO, P. O. Correção ionosférica utilizando os mapas globais do TEC do IGS: Avaliação no posicionamento por ponto na região brasileira. Boletim de Ciências Geodésicas v. 13, n. 2, p.253-270, jul-dez, 2007.

NOAA. National Geophysical Data Center. Disponível em: <http:/www.ngdc. noaa.gov/nndc/struts/form? $\mathrm{t}=102827 \& \mathrm{~s}=4 \& \mathrm{~d}=8,22,9>$, Acesso em: 10 de maio de 2012.

PETIT, G.; LUZUM, B. IERS Conventions (2010): IERS Technical Note $\mathrm{N}^{\circ}$ 36, Frankfurt am Main: Verlag des Bundesamts für Kartographie und Geodäsie, 2010. 179 p.

SEEBER, G. Satellite geodesy. $2^{\mathrm{a}}$ ed. Berlim: de Gruyter, 2003. p. 589.

SCHAER, S. Mapping and predicting Earth's ionosphere using the Global Positioning System. 1999, 205 p. Tese (Doutorado) - Naturwissenschaftçichen Fakultät. Universität Bern, Berna, 1999.

SCHAER, S.; GURTNER, W.; FELTENS, J. IONEX: The Ionosphere Map Exchange Format Version 1. In: IGS AC WORKSHOP, 1998, Darmstadt. Proceedings, 1998.

STONE, M. Cross-validation: a review. Statistics, v. 9, n. 1, p.- 127-139, 1978.

TULUNAY, E. et al. Forecasting total electron content maps by neural network technique, Radio Science, v. 41, RS4016, 2006.

WILAMOWSKI, B. M. et al. An algorithm for fast convergence in training neural networks. In: INTERNATIONAL JOINT CONFERENCE ON NEURAL NETWORKS. Proceedings..., 2001. p. 1778-1782.

WILAMOWSKI, B. M.; YU, H. Neural network learning without backpropagation. IEEE Transactions on Neural Networks, v. 21, n. 11, pp-1793-1803, Nov., 2010 .

(Recebido em setembro de 2012. Aceito em março de 2013). 